\title{
Growth and Functional Efficacy of Intrastriatal Nigral Transplants Depend on the Extent of Nigrostriatal Degeneration
}

\author{
Deniz Kirik, Christian Winkler, and Anders Björklund \\ Wallenberg Neuroscience Center, Department of Physiological Sciences, Lund University, 22184, Lund, Sweden
}

\begin{abstract}
Previous studies have shown that the functional efficacy of intrastriatal transplants of fetal dopamine (DA) neurons in the rat Parkinson model depends on their ability to establish a new functional innervation of the denervated striatum. Here we report that the survival, growth, and function of the grafted DA neurons greatly depend on the severity of the lesion of the host nigrostriatal system. Fiber outgrowth, and to a lesser extent also cell survival, were significantly reduced in animals in which part of the intrinsic DA system was left intact. Moreover, graftinduced functional recovery, as assessed in the stepping, pawuse, and apomorphine rotation tests, was obtained only in severely lesioned animals, i.e., in rats with $>70 \%$ DA denervation of the host striatum. Functional recovery seen in these
\end{abstract}

animals in which the 6-hydroxydopamine (6-OHDA) lesion was confined to the striatum was more pronounced than that previously obtained in rats with complete lesions of the mesencephalic DA system, indicating that spared portions of the host DA system, particularly those innervating nonstriatal forebrain areas, may be necessary for the grafts to exert their optimal functional effect. These data have implications for the optimal use of fetal nigral transplants in Parkinson patients in different stages of the disease.

Key words: Parkinson's disease; 6-hydroxydopamine; transplantation; sensorimotor behavior; stepping; paw use; tyrosine hydroxylase; stereology
Impairments in sensorimotor function induced by lesions of the nigrostriatal DA system can be reversed by intrastriatal transplants of nigral DA neurons taken from the fetal ventral mesencephalon (VM). However, functional recovery is incomplete: whereas some aspects of the DA deficiency syndrome are completely reversed (e.g., drug-induced turning behavior and sensorimotor neglect), other deficits, such as forelimb akinesia and skilled paw-use, are only partly or marginally affected by the grafted DA neurons (Björklund et al., 1994; Herman and Abrous, 1994; Winkler et al., 1999). The functional capacity of intrastriatal VM transplants is determined, at least in part, by the extent of striatal reinnervation provided by the grafted DA neurons and the placement of the cells, i.e., the subregion of the striatal complex reached by the graft-derived axons (Dunnett et al., 1983; Mandel et al., 1990; Annett et al., 1995). Nevertheless, attempts to improve the functional efficacy of intrastriatal nigral transplants by multiple graft placements have so far been unsuccessful (Nikkhah et al., 1993; Olsson et al., 1995; Winkler et al., 1999). In these experiments the entire caudate putamen was reinnervated to a level of $\sim 30-80 \%$ of normal, but recovery of forelimb akinesia and paw use remained incomplete. These studies, however, were performed in rats with a complete unilateral 6-OHDAinduced lesion of the medial forebrain bundle (MFB), resulting in a widespread denervation of both striatal, limbic, and cortical areas. The functional impairments seen in these animals, therefore, reflect a combination of striatal and nonstriatal dysfunction.

\footnotetext{
Received Dec. 4, 2000; revised Jan. 17, 2001; accepted Jan. 26, 2001.

This work was supported by grants from the Swedish Medical Research Council (04X-3874) and the Knut and Alice Wallenberg Foundation. We thank Ulla Jarl and Bengt Mattsson for expert technical assistance and Prof. O. Lindvall for critical reading of this manuscript.

Correspondence to should be addressed to D. Kirik, Wallenberg Neuroscience Center, Department of Physiological Sciences, Lund University, BMC A11, 22184, Lund, Sweden. E-mail: Deniz.Kirik@mphy.lu.se.

Copyright (C) 2001 Society for Neuroscience 0270-6474/01/212889-08\$15.00/0
}

The reinnervation provided by the grafts, by contrast, was limited to the striatum.

These observations raise the possibility that the functional efficacy of intrastriatal VM grafts may be limited by the extent of damage to the host DA system and that sparing of DA projections to nonstriatal forebrain targets may be essential for transplantinduced functional recovery. Here we report that the efficacy of intrastriatal nigral transplants is improved in rats with partial lesions of the nigrostriatal projection that leave the innervation of limbic and cortical areas intact. Moreover, we show that not only the extent of functional recovery but also the survival and axonal outgrowth of the grafted DA neurons are critically dependent on the severity of damage to the host DA system.

\section{MATERIALS AND METHODS}

A total of 39 young female Sprague Dawley rats (B\&K Universal, Stockholm, Sweden) were housed four or five to a cage with ad libitum access to rat chow and water under a $12 \mathrm{hr}$ light/dark cycle. All surgical operations were performed according to rules set by the Ethical Committee for use of Laboratory Animals at Lund University.

All surgical operations were performed using Equithesin ( $3 \mathrm{ml} / \mathrm{kg}$, i.p.) anesthesia. The animals were placed into stereotactic frames (Kopf Instruments). 6-OHDA injections were made using a $10 \mu \mathrm{l}$ Hamilton microsyringe. The injections of the VM cell suspensions were made with a $2 \mu$ l Hamilton microsyringe fitted with a glass pipette tip (outer diameter $60-80 \mu \mathrm{m}$ ). All anteroposterior (AP) and mediolateral (ML) coordinates were calculated from bregma, and dorsoventral (DV) coordinates were calculated from the dural surface. A small burr hole was made at the calculated coordinates. At the position of the entry of the glass pipette, a small cut in the dura was made using a 28 gauge stainless steel injection cannula. This prevents the glass pipette from bending and breaking during penetration. The injection rate was $1 \mu \mathrm{l} / \mathrm{min}$ for lesion and $0.3 \mu \mathrm{l} / \mathrm{min}$ for transplantation surgeries.

Thirty rats received a total of $28 \mu \mathrm{g}$ (free base) of 6-OHDA dissolved in $0.05 \%$ ascorbate-saline in four deposits in the right striatum as described (Kirik et al., 1998). They were then divided into two groups (severely and moderately impaired; each $n=15$ ) based on their stepping test scores. Each lesion group was further divided into two subgroups, 
Figure 1. Time course of surgery and testing. Changes in motor behavior were assessed in three sessions, 4-8 weeks after the lesion and 2-3 and 6-7 months after transplantation.

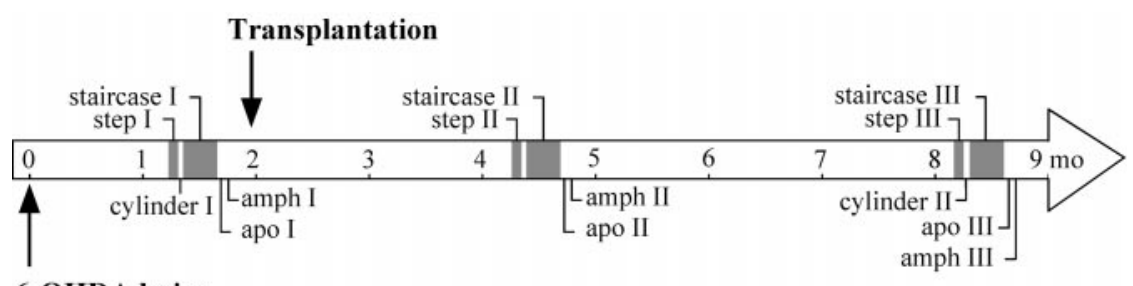

6-OHDA lesion one receiving transplants of VM cell suspension ( $n=8$ per group), the other sham surgery ( $n=7$ per group). Nine unoperated rats served as normal controls.

Two months after the lesion surgery, cell suspension grafts were prepared according to Nikkhah et al. (1994). VM tissue pieces from E14 embryos were incubated in $0.1 \%$ trypsin $/ 0.05 \%$ DNase in DMEM at $37^{\circ} \mathrm{C}$ for $20 \mathrm{~min}$, rinsed, mechanically dissociated to a single cell suspension, centrifuged, and resuspended to a concentration of $\approx 100,000$ cells $/ \mu$ l (viability $>97 \%$ ). A total of $\approx 450,000$ cells were distributed over five injection tracts in the lesioned striatum (AP: $+1.1,+0.6,+0.3,-0.5$, and -1.5 ; ML: $-3.4,-2.5,-3.8,-4.2$, and $-4.5 \mathrm{~mm}$ from bregma; tooth bar $=-3.3)$. Three $0.3 \mu \mathrm{l}$ deposits $(-5.3$ to $-3.3 \mathrm{~mm}$ below dura $)$ were delivered along each tract.

\section{Behavioral analysis}

Functional recovery in the lesioned and transplanted animals was monitored on a battery of tests (Fig. 1).

The stepping test (Schallert et al., 1992; Olsson et al., 1995) was performed at three time points during the experiment. At each time point the animals were tested twice daily on 3 consecutive days. Briefly, the rat was held by the experimenter fixing its hindlimbs with one hand and the forelimb not to be monitored with the other, while the unrestrained forepaw was touching the table. The number of adjusting steps was counted while the rat was moved sideways along the table surface ( 90 $\mathrm{cm}$ in $5 \mathrm{sec}$ ), in the forehand direction, (i.e., the animal is pulled to left when right paw is unrestrained), for both forelimbs.
The cylinder test (Schallert and Tillerson, 1999) was used to quantify forelimb use. The animals were videotaped as they moved freely in a 20 -cm-wide clear glass cylinder. Contacts made by each forepaw with the cylinder wall were scored from the videotapes by an observer blinded to the animals' identities. A total of 20 contacts were recorded for each animal, and the number of impaired (left) forelimb contacts as percentage of total formed the actual dependent variable.

Drug-induced rotation was assessed in automated rotometer bowls (Ungerstedt and Arbuthnott, 1970) after injection of apomorphine-HCl (Research Biochemicals Incorporated, St. Louis, MO; $0.25 \mathrm{mg} / \mathrm{kg}$, s.c., over $40 \mathrm{~min}$ ), or D-amphetamine sulfate (Apoteksbolaget, Lund, Sweden; $2.5 \mathrm{mg} / \mathrm{kg}$, i.p., over $90 \mathrm{~min}$ ). Data are expressed as net turns per minute with ipsilateral rotations assigned a positive value.

The staircase test (Montoya et al., 1991) was performed as described (Kirik et al., 1998). After $2 \mathrm{~d}$ of food deprivation the animals were placed in the test boxes baited bilaterally with 10 food pellets on each of the four steps $(45 \mathrm{mg})$. The animals were tested for $15 \mathrm{~min}$ on 7 consecutive days, until they reached a plateau performance. The number of pellets taken and the number of pellets eaten were counted.

\section{Histology}

Perfusion and tissue processing for histology: at 30 weeks after transplantation, the animals were anesthetized with pentobarbital and perfused through the ascending aorta with $50 \mathrm{ml}$ of isotonic saline, followed by $250 \mathrm{ml}$ of $4 \%$ paraformaldehyde in $0.1 \mathrm{M}$ phosphate buffer, post-fixed
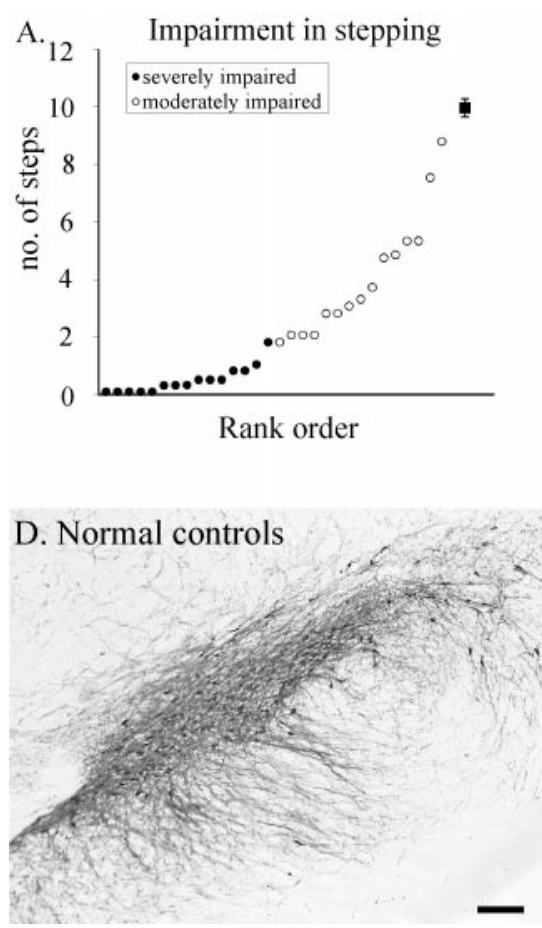

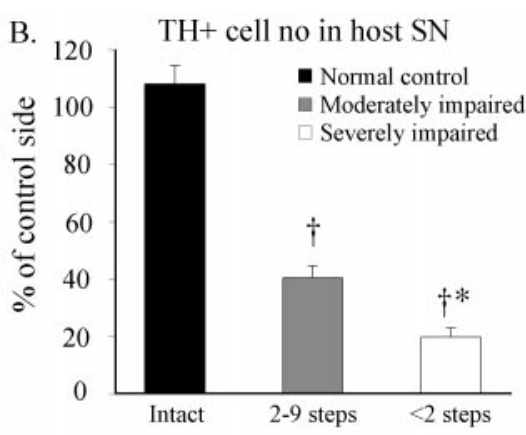

E. Moderately impaired

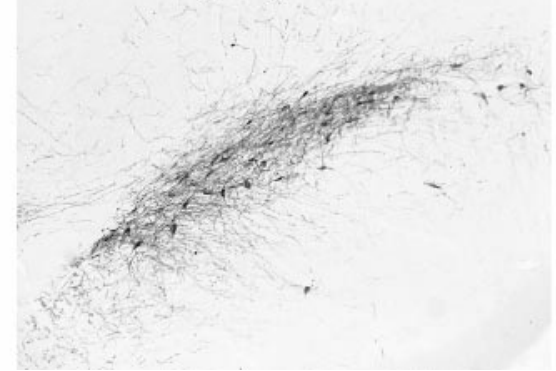

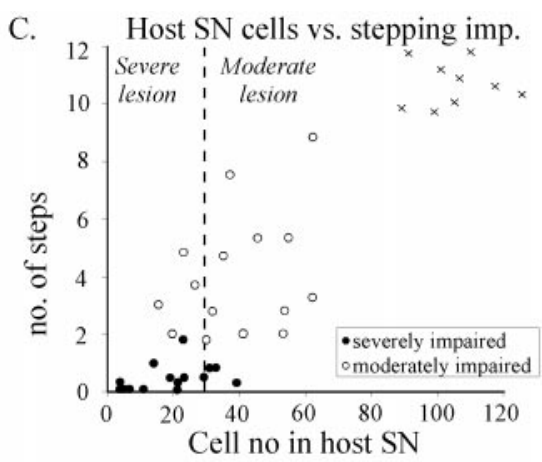

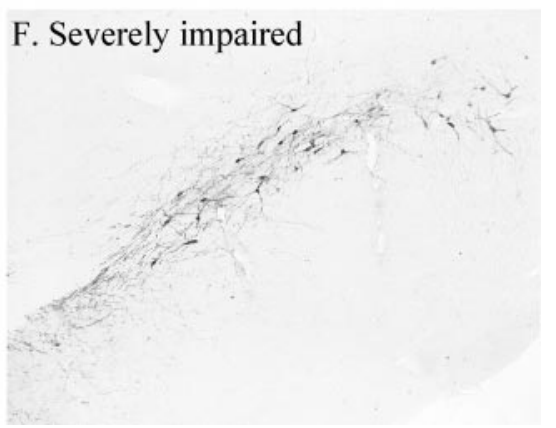

Figure 2. A, Based on the performance in the stepping test the animals were divided into severely impaired ( filled circles) and moderately impaired (open circles) groups (normal performance $=10-11$ steps; filled square). B, The loss of TH-positive cells in the SN was more pronounced in the severely impaired rats (ANOVA, $F_{(2,36)}=112.0, p<0.0001$; †different from normal controls, *different from moderately impaired). $C$, Impairment in the stepping test and extent of nigral cell loss were highly correlated $(r=0.86 ; p<0.0001)$. In the subsequent analysis $>70 \%$ cell loss is defined as severe lesion, and $45-70 \%$ cell loss is defined as a moderate lesion. $D-F$, Photomicrographs of sections through the central part of the SN stained for TH in normal control $(D)$, moderately impaired $(E)$, and severely impaired $(F)$ rats. Scale bar: $A, 0.2 \mathrm{~mm}$. 

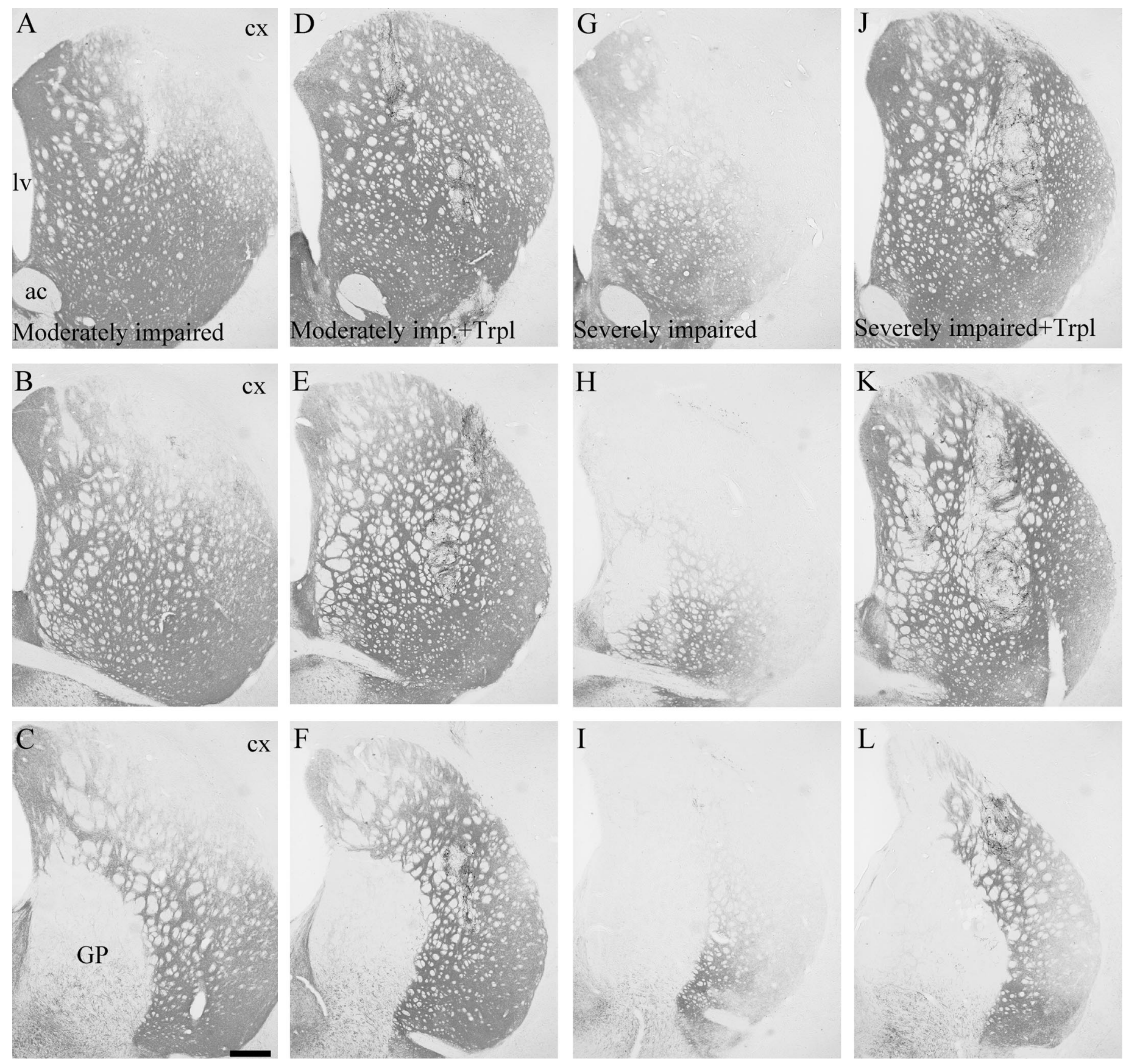

Figure 3. TH immunohistochemistry of striatal innervation. In the moderately impaired animals $(A-C)$ the striatal denervation was confined to the dorsolateral quadrant and extended more medially and ventrally in the severely impaired ones $(G-I)$. In transplanted animals $(D-F$ moderately impaired; $J-L$ severely impaired) the grafts had reinnervated sectors of the striatum that were depleted in the lesioned controls. The multiple graft deposits are distributed throughout the striatum: two in $D$, two in $E$, one in $F$, one in $J$, two in $K$, and one in $L . a c$, Anterior commissure; $c x$, neocortex; $G P$, globus pallidus; $l v$, lateral ventricle.

for $2 \mathrm{hr}$ in the same solution, and transferred to $20 \%$ sucrose before being sectioned on a freezing-stage microtome at $40 \mu \mathrm{m}$ into five series.

Immunohistochemical staining for tyrosine hydroxylase. The sections were rinsed three times in potassium-phosphate buffer (KPBS) between each incubation period. All incubation solutions contained $0.25 \%$ Triton $\mathrm{X}-100$ in KPBS. The sections were quenched for $10 \mathrm{~min}$ in $3 \% \mathrm{H}_{2} \mathrm{O}_{2} / 10 \%$ methanol in KPBS. Two hours of preincubation with 5\% normal horse serum (NHS) was followed by incubation with 1:2000 dilution of mouse anti-tyrosine hydroxylase (TH) antibody (Chemicon, Temecula, CA) in $2 \%$ NHS at room temperature. This was followed by incubation with 1:200 dilution of biotinylated horse anti-mouse antibody (BA2001; Vector Laboratories, Burlingame, CA) in $2 \%$ NHS. The secondary antibody step was followed with avidin-biotin-peroxidase complex (ABC Elite; Vector Laboratories). The reaction was visualized using 3,3diaminobenzidine as a chromogen. Sections were mounted on chromealum-coated slides, dehydrated in ascending alcohol concentrations, cleared in xylene, and coverslipped in DPX.

\section{Morphological analysis}

Total numbers of TH-positive cells were estimated with the optical fractionator (West et al., 1991), using the Olympus CAST-Grid system. Sections used for counting covered the entire substantia nigra (SN). The borders of the $\mathrm{SN}$ at all levels in the rostrocaudal axis were defined. The medial border was defined by a vertical line passing through the medial 
tip of the cerebral peduncle (and by the medial terminal nucleus of the accessory nucleus of the optic tract, when present in the sections), thereby excluding the TH-positive cells in the ventral tegmental area (VTA). Ventral border followed the dorsal border of the cerebral peduncle, thereby including the TH-positive cells in pars reticulata, and the area extended laterally to include the pars lateralis in addition to pars compacta. In the striatum all sections containing TH-positive cells were used to estimate total number of cells in the grafts.

Sampling was done using the Olympus CAST-Grid system (Olympus Denmark A/S). The system is composed of an Olympus BH2 microscope, an X-Y step motor stage run by an IBM personal computer, and a microcator (Heidenhain; ND 281). The computer-generated counting frame was placed randomly on the first counting area and systematically moved through all fields at $100 \times$ oil objective. Only the profiles that came into focus within the counting volume were counted. The estimate of the total number of neurons was calculated according to the optical fractionator formula (for more details, see West et al. (1991). The coefficient of error was calculated according to Gundersen and Jensen (1987), and values $<0.10$ were accepted.

Striatal fiber density measurements. The optical densities of the THimmunoreactive fibers in the striatum were measured using the National Institutes of Health 1.62 Image program on a Macintosh 9500 computer connected to a digital camera (ProgRes; Kontron Elektronik) and a constant illumination table. For each animal the optical density was measured at seven rostrocaudal levels according to the atlas of including either the entire cross-sectional area of the striatum (excluding the grafts) or the dorsolateral sector: (1) AP, +1.6; (2) AP, +1.0; (2) AP, +0.2 ; (3) AP, -0.3 ; (4) AP, -0.9 ; (5) AP, -1.4 ; and (7) AP, $-2.1 \mathrm{~mm}$ relative to bregma. The dorsolateral sector was demarcated by the mid dorsoventral and the mid mediolateral lines that divide the striatum into four equal quadrants at each level. Readings were corrected for nonspecific background density, as measured from the completely denervated parts of the striatum and presented as percentage of the intact side.

Statistical analysis. Group comparisons used ANOVA, or repeated measures ANOVA when necessary, followed by post hoc analysis using Student-Newman-Keuls test (significance set to 5\%). Correlations were done using simple linear regression analysis.

\section{RESULTS}

The 6-OHDA-lesioned rats were allocated to two groups based on their performance in the stepping test. The first 15 animals in the rank order formed the "severely impaired animals" (Fig. 2A, filled circles) making less than two steps with the paw contralateral to the lesion in the stepping test; and the rest $(n=15)$ were allocated as "moderately impaired animals" (Fig. $2 A$, open circles) making two to nine steps. Normal performance in the control group was $10-11$ steps $(n=9)$ (Fig. $2 A$, filled square, $C$, crosses). The variability in the stepping test was reflected in the extent of cell loss in the ipsilateral SN (Fig. 2E,F). Loss of $\mathrm{TH}$-positive cells in $\mathrm{SN}$ was $\sim 60 \%$ in the moderately impaired animals and $>80 \%$ in the severely impaired ones (Fig. $2 B$ ). The overall correlation between the rats' performance in the stepping test and the TH-positive cell numbers in the $\mathrm{SN}$ was highly significant (Fig. 2C). To determine the effect of lesion severity on graft survival and fiber outgrowth, the animals were allocated in a "severely lesioned" ( $>70 \%$ loss of TH-positive cells in $\mathrm{SN}$ ) and a "moderately lesioned" group (45-70\% cell loss) (Fig. 2C, dashed line). Cell loss in VTA was $<20 \%$ in all animals (data not shown).

In the moderately impaired animals the extensive denervation in the striatum was limited to the dorsal and lateral sectors throughout the rostrocaudal extent of the caudate putamen with more diff use lower intensity in the other parts (Fig. $3 A-C$ ). The density of the remaining striatal $\mathrm{TH}$-positive fiber innervation measured from the entire striatum averaged $50 \%$ of intact values (Fig. 4). In the severely impaired animals, the denervation was more substantial with an overall reduction in the whole striatal TH-positive innervation of $\sim 80 \%$ (Fig. 4). The spared fibers were confined to the most medial and ventral parts (Fig. $3 G-I$ ). The

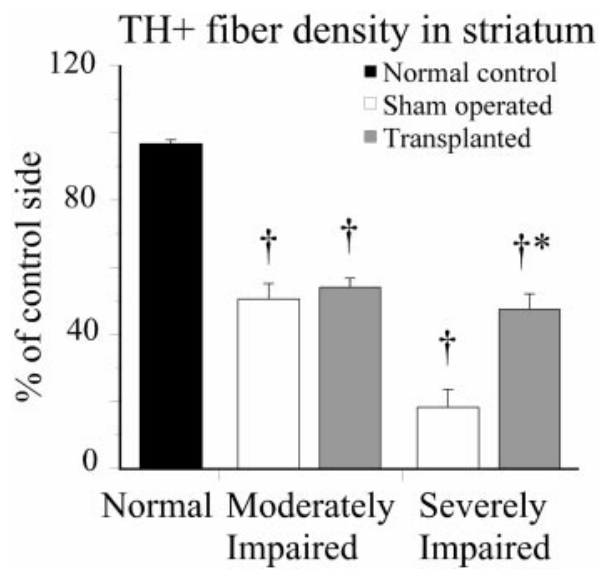

Figure 4. The overall striatal TH-positive fiber density was improved by the graft only in the severely impaired animals (ANOVA, $F_{(4,34)}=58.4$, $p<0.0001$; *different from its sham-operated controls, †different from normal controls). Scale bar: $C, 0.5 \mathrm{~mm}$.

innervation of the nucleus accumbens, olfactory tubercle, septum, and amygdala was unaffected in all animals. When all shamoperated animals and normal controls are considered, there was a highly significant correlation between the extent of behavioral impairment, as assessed in the stepping, staircase, and rotation tests, and the overall reduction in the TH-positive innervation of the striatum (Fig. 5).

\section{Survival and growth of the grafted DA neurons}

The VM cell suspension grafts survived in all animals $(n=16)$, as assessed by $\mathrm{TH}$ immunohistochemistry (Fig. 3D-F,J-L). The total number of surviving TH-positive cells in the striatum varied between 3108 and 15,155. As seen in the scatterplot in Figure $6 \mathrm{~A}$ there was an overall trend toward poorer graft survival in animals with more restricted degeneration of the intrinsic nigrostriatal DA projection, although not statistically significant $(r=0.47 ; p=$ $0.064)$. However, when the animals were grouped based on the extent of TH-positive cell loss in the host SN (Fig. 2C), the grafts in the severely lesioned rats showed significantly higher numbers of surviving TH-positive neurons compared with the grafts in animals with moderate lesions (5527 \pm 896 and $9190 \pm 1394$, respectively) (Fig. 6B). This corresponds to a survival rate of the TH-positive cells of $\sim 10-20 \%$ in the grafting procedure.

The TH-positive fibers derived from the graft were able to reinnervate the sectors of the striatum that were initially denervated by the 6-OHDA lesion, both in the moderately and in the severely impaired animals (Fig. 3, compare $A-C$ with $D-F$ and $G-I$ with $J-L)$. However, in neither group did the density of striatal TH-positive fiber innervation reach normal levels (Fig. 4). In the severely impaired, transplanted animals the overall striatal TH-positive fiber innervation increased from $\sim 20-50 \%$ of normal (Fig. 4), and within the most denervated dorsolateral sector from $<5 \%$ to between 40 and $60 \%$. In the moderately impaired animals the overall mean striatal TH-positive fiber density (all striatal levels combined) was similar in the transplanted and the sham-operated rats (Fig. 3S). However, within the denervated dorsolateral sector the grafts had restored TH-positive fiber density to $\sim 40-50 \%$ of normal, compared with $20-25 \%$ of normal in the sham-operated control rats. In the less denervated ventral striatum the innervation density, $50-90 \%$ of normal, was similar in both grafted and nongrafted rats.

These data indicate that the magnitude of graft-derived fiber 
A. Left Forelimb Stepping

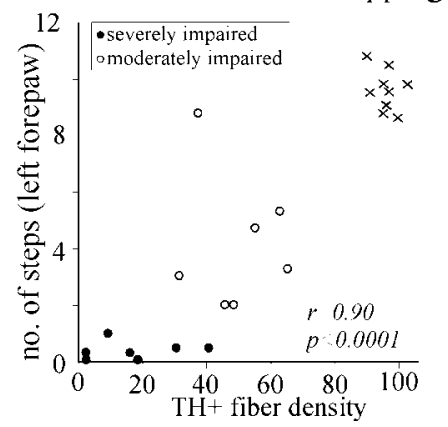

B. Staircase Test

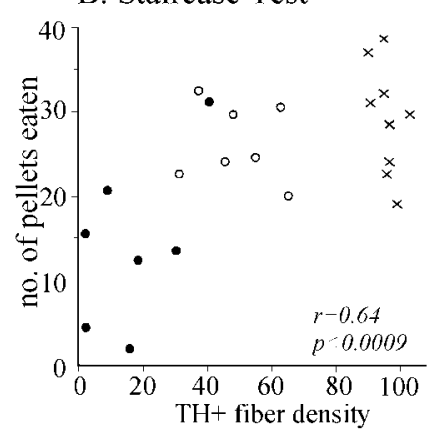

C. Amphetamine rotation

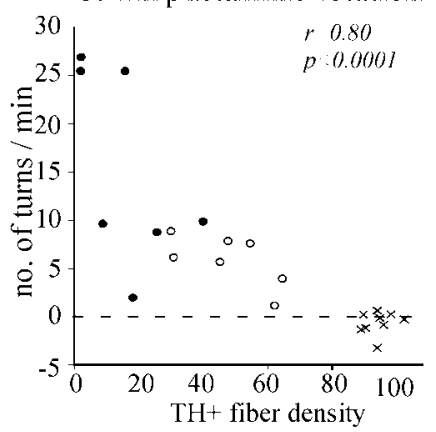

D. Apomorphine rotation

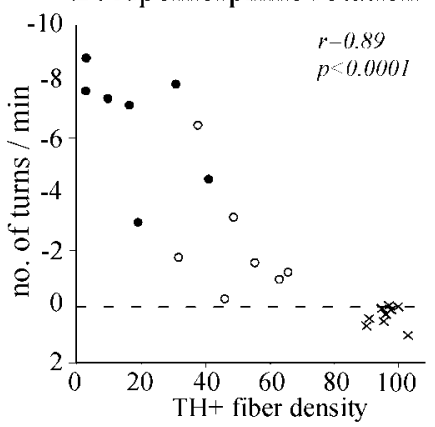

Figure 5. Linear correlation between TH-fiber density in the striatum and performance in different tests of motor behavior as shown for the stepping $(A)$, staircase $(B)$, amphetamine rotation $(C)$, and apomorphine rotation $(D)$ tests. The highest correlation were seen for forelimb stepping $(r=0.90)$ and apomorphine rotation $(r=0.89)$, indicating that these two tests have the best predictive value.
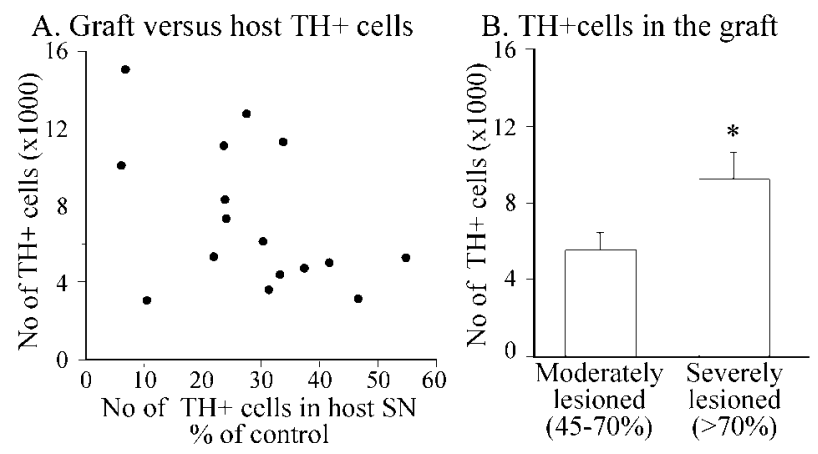

Figure 6. A, The correlation between the number of TH-positive cells in host $\mathrm{SN}$ and the number of surviving $\mathrm{TH}$-positive cells in the graft was overall nonsignificant $(r=0.47 ; p=0.064)$. $B$, However, the survival of the grafted TH-positive neurons was significantly greater in the severely lesioned animals (*different from moderate lesions; unpaired $t$ test, $p<0.05)$.

outgrowth depended on the extent of denervation in the host striatum. In Figure $7 A$ the total striatal innervation density in the individual transplant and control rats (all striatal levels combined) is plotted against the severity of the lesion as assessed by the magnitude of cell loss in the host SN. The convergence of the two regression lines, for animals with transplants (solid line and filled circles) and for the sham-operated controls (dashed line and open circles) indicate that the total fiber outgrowth is greatly reduced in animals with smaller lesions. Indeed, the overall graftderived fiber outgrowth in rats with less severe (45-70\%) lesions was only half of that in rats with severe (70-97\%) lesions, and only one-third of that previously seen in rats with complete $(>97 \%)$ lesions of the nigrostriatal system having similar size grafts (Fig. 7B) (cf. Winkler et al., 1999).

\section{Impact of VM transplants on motor behavior}

In the pretransplant test the lesioned animals showed a wide range of impairments in motor function on the side contralateral to the 6-OHDA lesion. The animals defined as severely impaired in the stepping test (Fig. $8 A$ ) were significantly impaired also in paw use in the cylinder test (Fig. $8 B$ ), in paw reaching in the staircase test (ANOVA, $F_{(4,34)}=5.9, p=0.001$; data not shown), and in turning behavior in the rotation tests (Fig. 8C,D). These deficits remained stable over time in the sham-operated control group, except in the staircase test in which the animals improved their performance in the second and third tests to the level seen in the intact animals (ANOVA effect of time; $F_{(2,12)}=4.9, p=$
0.03, data not shown). The animals defined as moderately impaired in the stepping test showed a moderate amphetamine and apomorphine-induced turning response, a marginal impairment in the cylinder test, but no impairment in the staircase test (Fig. $8 A-C)$.

In the severely impaired animals transplant-induced functional recovery was observed in both spontaneous and drug-induced motor behavior (Fig. 8). In the stepping test the grafted animals improved from $0.5 \pm 0.2$ steps before, to $4.5 \pm 1.0$ steps at 7 months after transplantation (ANOVA effect of time, $F_{(2,14)}=$ $12 ; p<0.001$ ), i.e., from $<10 \%$ to $\sim 50 \%$ of control performance (Fig. 8A), and in the cylinder test left touches increased from $27.5 \pm 4.5 \%$ to $40.7 \pm 5.1 \%$ at 7 months, which is close to normal symmetric paw use (Fig. $8 B$ ). In both tests the grafted animals were also significantly improved over their sham-operated controls. Turning behavior was reduced by $60-80 \%$ at 3 and 7 months after transplantation in the apomorphine test (Fig. 8D), and overcompensated, i.e., reversed to the contralateral direction, in the amphetamine test (Fig. $8 C$ ). Recovery was observed also in the paw reaching test but did not differ from that seen in the sham-operated controls (ANOVA group $\times$ time interaction, $\left.F_{(8,68)}=0.98, p=0.46\right)$.

In the moderately impaired animals a transplant-induced effect was seen in amphetamine-induced rotation (Fig. 8C), but in none of the other tests. In the stepping test all animals showed some spontaneous recovery over time (ANOVA effect of time, $F_{(2,12)}=$ $6.03, p=0.015)$, but the grafted animals did not differ from the sham-operated controls. At 7 months after transplantation both groups remained significantly impaired compared with the intact animals. Paw use in the cylinder test, which was not significantly affected by the transplant (Fig. $8 B$ ), and the low rate of apomorphine-induced rotation (1-2 turns/min) remained unchanged.

\section{DISCUSSION}

The results show that the survival, growth, and functional efficacy of intrastriatal VM transplants depend on the severity of the lesion of the host nigrostriatal system, i.e., the extent of DA denervation of the host striatum. In three of the behavioral tests (stepping, cylinder, and apomorphine rotation tests) graftinduced improvement was seen only in the severely impaired animals, i.e., in rats with $>70 \%$ denervation of the lesioned striatum. In animals with less severe lesions (40-70\% striatal denervation) identical transplants had no effect. This difference in functional efficacy is readily explained by differences in DA 
Figure 7. A, Nigral TH-positive cell number and striatal TH-positive innervation were highly correlated in the sham-operated and normal controls (dashed line; $r=0.93$, $p<0.0001)$, as well as in the grafted animals $(r=0.62$; $p=0.01)$. $B$, The estimated graft-derived TH-positive fiber density for the severely lesioned ( $>70 \%$ cell loss) and moderately lesioned rats $(45-70 \%$ cell loss) were generated by calculating the difference between the individual density values of the grafted animals (A, filled circles) and the regression line derived from the lesioned animals (dashed line). The data for complete lesions are from Winkler et al. (1999).
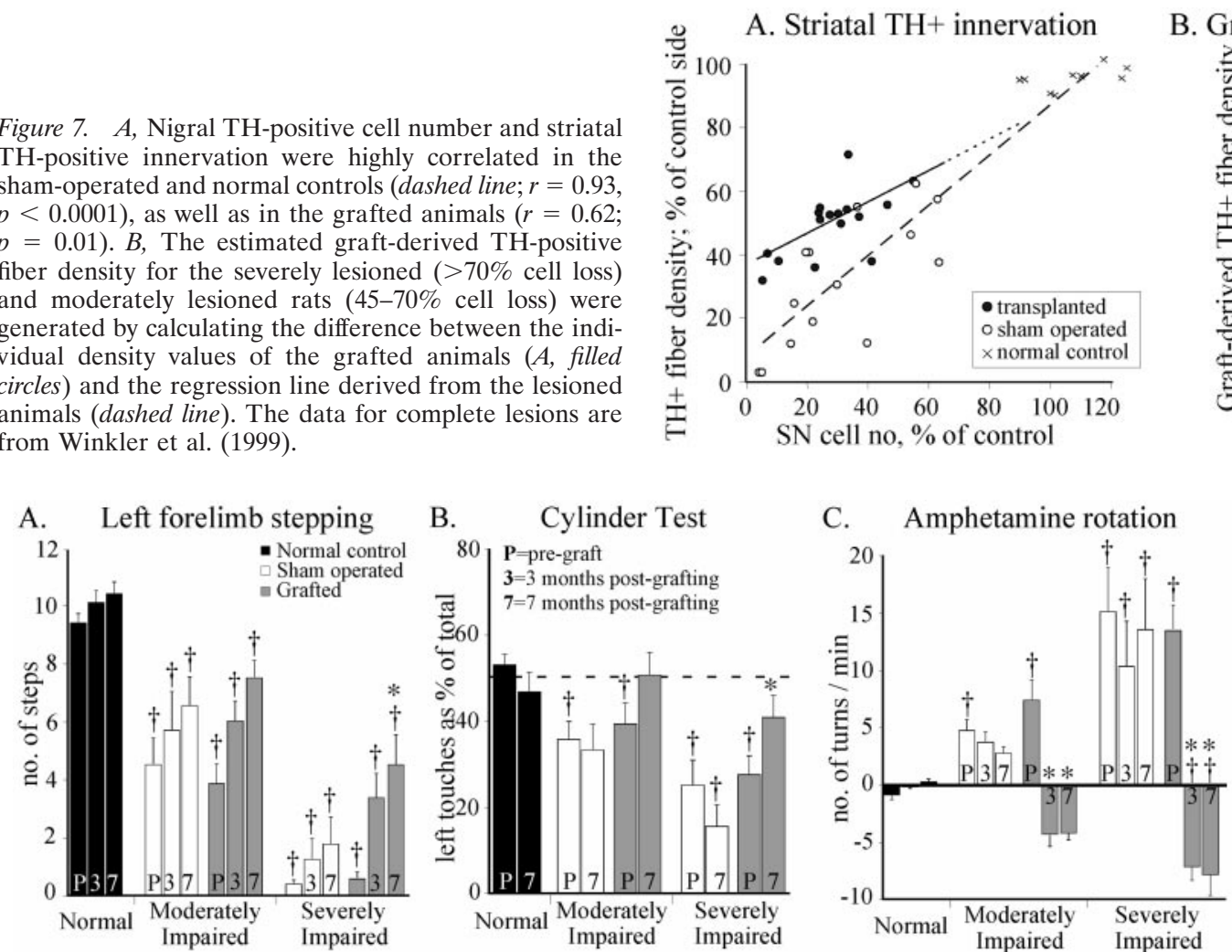

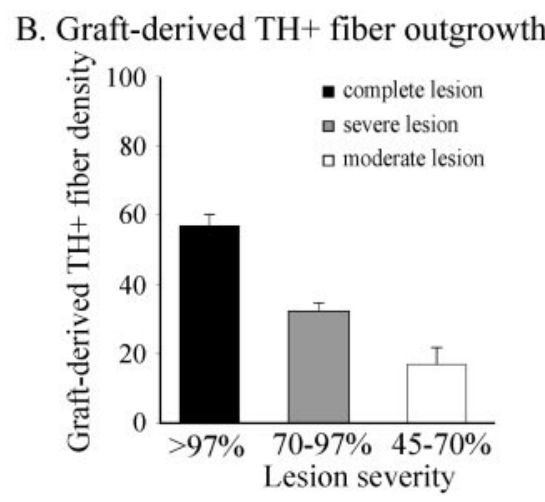

Figure 8. Lesion-induced behavioral impairments were assessed at three time points: pregrafting (designated as $P$ ) and 3 and 7 months after grafting. The severely impaired group showed significant recovery in the stepping test $\left(A\right.$, ANOVA, $\left.F_{(4,34)}=17.4, p<0.0001\right)$, the cylinder test $(B$, ANOVA, $\left.F_{(4,34)}=6.9, p<0.001\right)$, amphetamine $\left(C\right.$, ANOVA, $\left.F_{(4,34)}=15.8, p<0.0001\right)$, and apomorphine rotation $\left(D\right.$, ANOVA, $\left.F_{(4,34)}=15.2, p<0.0001\right)$. The moderately impaired, transplanted animals showed significant changes only in amphetamine rotation. *Different from sham-operated control; $\uparrow$ different from normal control; $p<0.05$.

neuron survival and fiber outgrowth from the VM transplants. In the severely lesioned animals the survival of TH-positive neurons was increased by an average of $70 \%$, and graft-derived $\mathrm{TH}-$ positive fiber outgrowth by an average of $100 \%$, compared with grafts in animals with more restricted $(<70 \%)$ striatal denervation. This effect of lesion severity is further pronounced in animals with complete $(>97 \%)$ striatal denervation, having grafts of the same size, showing a threefold greater graft-derived THpositive fiber outgrowth than in the moderately lesioned animals studied here (Fig. 7B) (cf. Winkler et al., 1999).

These data indicate that the grafted DA neurons interact with the striatal target in two ways. First, the denervated striatal target provides a positive stimulatory effect on the outgrowth of axons, and to a lesser extent also on the survival of the grafted cells, and, second, that this stimulatory effect is reduced in rats in which part of the intrinsic DA system is left intact. Indeed, in the partially denervated striatum, the graft-derived reinnervation was largely restricted to the severely denervated areas, and the total striatal DA innervation density, i.e., the residual innervation from the host and the graft-derived innervation combined, did not exceed $60-70 \%$ of normal in any animal. This suggests that the ability of the denervated striatum to promote further fiber outgrowth is lost at this level of innervation density (illustrated by the converging regression lines for the grafted and sham-operated animals in Fig. 7A).

Although the intrastriatal 6-OHDA lesion model is now well established, the long-term assessment of behavioral functions and its correlation to the quantitative morphological evaluation has not been studied in detail. It is notable that the sham-operated animals in this study showed a general tendency to partially recover in the stepping test while the cylinder test, or the druginduced rotations were not changed. This recovery was indeed significant in the moderately impaired animals. The failure of the grafts to induce any significant behavioral effect in the moderately impaired rats may also, in part, be attributable to the spontaneous recovery that took place over time in these groups of animals. Whether, this recovery is a result of a gradual regeneration from the remaining fibers leading to reinnervation of initially depleted areas is at present unknown.

Previous studies have shown that the DA-denervated striatum exerts a growth-stimulating effect on grafted nigral DA neurons (Doucet et al., 1990). When given a choice between striatal and nonstriatal targets, grafted nigral DA neurons will grow axons selectively into the denervated striatum (Björklund et al., 1983; Wictorin et al., 1992), and DA neurons placed in a non-DAinnervated brain region extend axons within the graft, but not into the surrounding host tissue (Björklund et al., 1983). Both cell-specific recognition molecules and diffusible growthstimulating factors may be involved in this effect. Carvey et al. (1989, 1996) have shown that adult striatal tissue contains a neurotrophic activity (active on DA neurons in culture) that is increased after lesion of the nigrostriatal afferents or blockade of DA receptors. The factor or factors involved have so far not been identified, but several growth factors with neurotrophic activity on DA neurons are known to be expressed at elevated levels in the DA-denervated striatum (Funa et al., 1996; Zhou et al., 1996; 
Yurek and Fletcher-Turner, 2000), and delivery of such factors (bFGF, BDNF, and GDNF, in particular) by engineered cells or intrastriatal delivery, have been shown to enhance both survival and outgrowth from intrastriatal VM transplants (Takayama et al., 1995; Rosenblad et al., 1996; Yurek et al., 1996; Wilby et al., 1999). For one of these factors, BDNF, there are data to indicate that the magnitude of the denervation-induced increase in the striatum is directly correlated with the severity of the 6-OHDA lesion (as determined by the amphetamine rotation score) (Yurek and Fletcher-Turner, 2000). Interestingly, Collier et al. (1999) have recently reported that the ability of the denervated striatum to promote fiber outgrowth from VM transplants declines with age of the host. This is consistent with observations of other investigators showing that striatal neurotrophic activity declines with age (Ling et al., 2000) and that the denervation-induced increase in BDNF in the striatum is significantly reduced in aged rats (Yurek and Fletcher-Turner, 2000). Diffusible growthpromoting factors, including BDNF, are thus possible candidates for the mediation of the lesion-dependent target derived neurotrophic effect.

The magnitude of transplant-induced functional recovery obtained in rats with severe partial lesions was greater than that previously obtained with similar sized grafts in rats with complete MFB lesions (Winkler et al., 1999). In rats with complete lesions of the mesencephalic DA projection, which denervate not only the striatum but also nucleus accumbens, olfactory tubercle, and associated limbic and cortical forebrain areas, there is a clear mismatch between the density of TH-positive fibers in the reinnervated striatum (reaching 70-90\% of normal in the most densely innervated parts) and the relatively poor performance in the forelimb akinesia test [a maximum of 3.5 steps in the two experiments reported by Winkler et al. (1999)]. In the present experiment, by contrast, the level of performance regained in the grafted animals (mean of five steps; i.e., $\sim 50 \%$ of control) matches the level of striatal TH-positive fiber density in the grafted striatum, which was $\sim 50 \%$ of normal. As illustrated in Figure 9 this corresponds to the level of performance seen in rats with similar size partial lesions of the intrinsic nigrostriatal system (Kirik et al., 1998), whereas the recovery seen in rats with complete MFB lesions is clearly below the expected level (Fig. 9, filled diamond).

These data indicate that the more extensive forebrain denervation in animals with complete MFB lesions may compromise the overall functional impact of intrastriatal VM transplants. Spared portions of the mesencephalic DA projection system, particularly those innervating nonstriatal forebrain areas, may thus be necessary for the intrastriatal VM grafts to exert their optimal functional effects. The mesolimbocortical DA system is known to play an important role in motor behavior, such as response selection, behavioral switching, and incentive-motivational processes, and execution of coherent behavioral responses is likely to require coordinated DA release in both striatal and nonstriatal areas (Dunnett and Robbins, 1992). Reinnervation of the dorsal striatum alone, therefore, in the absence of a functional mesolimbocortical DA pathway, may be insufficient to normalize motor behavior in 6-OHDA-lesioned rats.

In conclusion, the results show that the functional efficacy of intrastriatal VM transplants is greatly dependent on the severity of damage to the host nigrostriatal system, and that this works in two ways. More restricted damage of the striatal DA innervation reduces survival and growth of the grafted neurons, and more extensive near-complete lesions reduces the functional impact of

\section{Stepping 7 month after grafting}

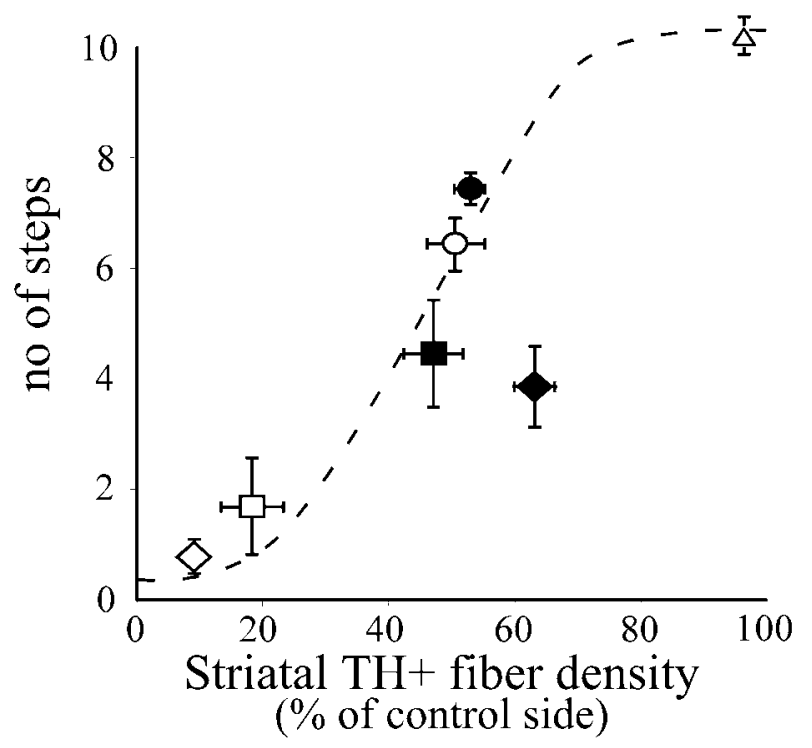

Figure 9. The correlation between lesion severity and the stepping test fits to an S-shaped curve (Kirik et al., 1998). The performance of the different groups (mean \pm SEM) at 7 months after grafting is plotted on the graph to demonstrate the efficacy of the transplants in the severely impaired, grafted group (filled square) and the moderately impaired, grafted group ( filled circles), compared with the severely impaired (open squares) and the moderately impaired, sham-operated animals (open circles). Filled and open diamonds [data from Winkler et al. (1999)] give the performance of completely lesioned animals with or without grafts, respectively.

otherwise effective grafts. These observations have implications of principal importance for cell transplantation in Parkinson's disease. First, PD patients have, to a varying degree, reductions in DA not only in caudate nucleus or the putamen but also in several extrastriatal cortical and subcortical forebrain areas (Scatton et al., 1983; Agid et al., 1987). Patients in advanced stages of the disease, therefore, may be less suitable candidates if the disease has progressed to involve also nonstriatal areas. This may be possible to determine by PET scanning before grafting. Similarly, the efficacy of an established functional graft may be lost over time in case the DA innervation of nonstriatal areas continues to deteriorate. Second, in patients with early disease, the ability of the partially denervated striatum to sustain survival and growth of the grafted neurons may be reduced. The lack of target-derived trophic support at intermediate levels of striatal denervation may thus limit the functional efficacy of intrastriatal nigral grafts. Supply of neurotrophic factors, e.g., GDNF or BDNF, may be used to obtain the combined effect of blocking further deterioration of the intrinsic DA system and improving graft survival and function, i.e., by providing an increased and long-lasting growth stimulus for the grafted neurons that simulates the effect of striatal denervation.

\section{REFERENCES}

Agid F, Javoy-Agid F, Ruberg M (1987) Biochemistry of neurotransmitters in Parkinson's disease. In: Movement disorders (Marsden CD, Fahn S, eds), pp 166-230. Stoneham, MA: Butterworth.

Annett L, Torres E, Ridley R, Baker H, Dunnett S (1995) A comparison of the behavioural effects of embryonic nigral grafts in the caudate nucleus and in the putamen of marmosets with unilateral 6-OHDA lesions. Exp Brain Res 103:335-371.

Björklund A, Stenevi U, Schmidt RH, Dunnett SB, Gage FH (1983) 
Intracerebral grafting of neuronal cell suspensions. II. Survival and growth of nigral cell suspensions implanted in different brain sites. Acta Physiol Scand [Suppl] 522:9-18.

Björklund A, Dunnett SB, Nikkhah G (1994) Nigral transplants in the rat Parkinson model. Functional limitations and strategies to enhance nigrostriatal reconstruction. In: Functional neural transplantation (Dunnett SB, Björklund A, eds), pp 47-68. New York: Raven.

Carvey PM, Ptak LR, Kao L, Klawans HL (1989) Striatal homogenates from animals chronically treated with haloperidol stimulate dopamine and GABA uptake in cultures of rostral mesencephalic tegmentum. Clin Neuropharmacol 12:425-434.

Carvey PM, Lin DH, Faselis CJ, Notermann JK, Ling ZD (1996) Loss of striatal DA innervation increases striatal trophic activity directed at DA neurons in culture. Exp Neurol 140:184-197.

Collier TJ, Sortwell CE, Daley BF (1999) Diminished viability, growth, and behavioral efficacy of fetal dopamine neuron grafts in aging rats with long-term dopamine depletion: an argument for neurotrophic supplementation. J Neurosci 19:5563-5573.

Doucet G, Brundin P, Descarries L, Björklund A (1990) Effect of prior dopamine denervation on survival and fiber outgrowth from intrastriatal fetal mesencephalic grafts. Eur J Neurosci 2:279-290.

Dunnett SB, Robbins TW (1992) The functional role of mesotelencephalic dopamine systems. Biol Rev Camb Philos Soc 67:491-518.

Dunnett SB, Björklund A, Schmidt RH, Stenevi U, Iversen SD (1983) Intracerebral grafting of neuronal cell suspensions. IV. Behavioural recovery in rats with unilateral 6-OHDA lesions following implantation of nigral cell suspensions in different forebrain sites. Acta Physiol Scand [Suppl] 522:29-37.

Funa K, Yamada N, Brodin G, Pietz K, Ahgren A, Wictorin K, Lindvall O, Odin P (1996) Enhanced synthesis of platelet-derived growth factor following injury induced by 6-hydroxydopamine in rat brain. Neuroscience 74:825-833.

Gundersen HJG, Jensen EB (1987) The efficiency of systematic sampling in stereology and its prediction. J Microsc 147:229-263.

Herman JP, Abrous ND (1994) Dopaminergic neural grafts after fifteen years: results and perspectives. Prog Neurobiol 44:1-35.

Kirik D, Rosenblad C, Björklund A (1998) Characterization of behavioral and neurodegenerative changes following partial lesions of the nigrostriatal dopamine system induced by intrastriatal 6-hydroxydopamine in the rat. Exp Neurol 152:259-277.

Ling ZD, Collier TJ, Sortwell CE, Lipton JW, Vu TQ, Robie HC, Carvey PM (2000) Striatal trophic activity is reduced in the aged rat brain. Brain Res 856:301-309.

Mandel RJ, Brundin P, Björklund A (1990) The importance of graft placement and task complexity for transplant-induced recovery of simple and complex sensorimotor deficits in dopamine denervated rats. Eur J Neurosci 2:888-894.

Montoya CP, Campbell HL, Pemberton KD, Dunnett SB (1991) The "staircase test": a measure of independent forelimb reaching and grasping abilities in rats. J Neurosci Methods 36:219-228.

Nikkhah G, Duan WM, Knappe U, Jödicke A, Björklund A (1993) Restoration of complex sensorimotor behavior and skilled forelimb use by a modified nigral cell suspension transplantation approach in the rat Parkinson model. Neuroscience 56:33-43.
Nikkhah G, Olsson M, Eberhard J, Bentlage C, Cunningham MG, Björklund A (1994) A microtransplantation approach for cell suspension grafting in the rat Parkinson model: a detailed account of the methodology. Neuroscience 63:57-72.

Olsson M, Nikkhah G, Bentlage C, Björklund A (1995) Forelimb akinesia in the rat Parkinson model: differential effects of dopamine agonists and nigral transplants as assessed by a new stepping test. J Neurosci 15:3863-3875.

Rosenblad C, Martinez-Serrano A, Björklund A (1996) Glial cell linederived neurotrophic factor increases survival, growth and function of intrastriatal fetal nigral dopaminergic grafts. Neuroscience 75:979-985.

Scatton B, Javoy-Agid F, Rouquier L, Dubois B, Agid Y (1983) Reduction of cortical dopamine, noradrenaline, serotonin and their metabolites in Parkinson's disease. Brain Res 275:321-328.

Schallert T, Tillerson JL (1999) Intervention strategies for degeneration of dopamine neurons in Parkinsonism: optimising behavioral assessment of outcome. In: Innovative models of CNS disease: from molecule to therapy. Clifton, NJ: Humana.

Schallert T, Norton D, Jones TA (1992) A clinically relevant unilateral rat model of Parkinsonian akinesia. J Neural Transpl Plast 3:332-333.

Takayama H, Ray J, Raymon HK, Baird A, Hogg J, Fisher LJ, Gage FH (1995) Basic fibroblast growth factor increases dopaminergic graft survival and function in a rat model of Parkinson's disease. Nat Med $1: 53-58$

Ungerstedt U, Arbuthnott G (1970) Quantitative recording of rotational behavior in rats after 6-hydroxydopamine lesions of the nigrostriatal dopamine system. Brain Res 24:485-493.

West MJ, Slomianka L, Gundersen HJG (1991) Unbiased stereological estimation of the total number of neurons of the rat hippocampus using the optical fractionator. Anat Rec 231:482-497.

Wictorin K, Brundin P, Sauer H, Lindvall O, Björklund A (1992) Long distance directed axonal growth from human dopaminergic mesencephalic neuroblasts implanted along the nigrostriatal pathway in 6-hydroxydopamine lesioned adult rats. J Comp Neurol 323:475-494.

Wilby MJ, Sinclair SR, Muir EM, Zietlow R, Adcock KH, Horellou P, Rogers JH, Dunnett SB, Fawcett JW (1999) A glial cell line-derived neurotrophic factor-secreting clone of the Schwann cell line SCTM41 enhances survival and fiber outgrowth from embryonic nigral neurons grafted to the striatum and to the lesioned substantia nigra. J Neurosci 19:2301-2312.

Winkler C, Bentlage C, Nikkhah G, Samii M, Björklund A (1999) Intranigral transplants of GABA-rich striatal tissue induce behavioral recovery in the rat Parkinson model and promote the effects obtained by intrastriatal dopaminergic transplants. Exp Neurol 155:165-186.

Yurek DM, Fletcher-Turner A (2000) Lesion-induced increase of BDNF is greater in the striatum of young versus old rat brain. Exp Neurol 161:392-396.

Yurek DM, Lu W, Hipkens S, Wiegand SJ (1996) BDNF enhances the functional reinnervation of the striatum by grafted fetal dopamine neurons. Exp Neurol 137:105-118.

Zhou J, Pliego-Rivero B, Bradford HF, Stern GM (1996) The BDNF content of postnatal and adult rat brain: the effects of 6-hydroxydopamine lesions in adult brain. Brain Res Dev Brain Res 97:297-303. 